\section{A Double Hard Sphere Model of Supercooled Liquid Gallium}

\author{
B. R. Orton
}

Physics Department, Brunel University, Uxbridge, U.K.

$$
\begin{gathered}
\text { (Z. Naturforsch. } 32 \text { a, } 332-334 \text { [1977]; } \\
\text { received January } 3,1977 \text { ) }
\end{gathered}
$$

A double hard sphere model, already successfully applied to liquid $\mathrm{Ge}$ and $\mathrm{Sb}$, has been used to reproduce the interference function of supercooled liquid $\mathrm{Ga}$ at $23{ }^{\circ} \mathrm{C}$. The model interference function was composed of two partial interference functions which corresponded to packing densities of 0.50 and 0.47 and diameters $2.78 \AA$ and $2.25 \AA$. Additional justification for the model has been found in recent theoretical work.

\section{Introduction}

In the solid state there are several possible forms of the structure of $\mathrm{Ga}^{1}$. $\mathrm{Ga}(\mathrm{I})$ is most relevant to the present work because it is stable at normal pressures and temperatures, while the other forms are not. $\mathrm{Ga}(\mathrm{I})$ has an orthorhombic unit cell and each atom has seven near neighbours, one at the short distance of $2.44 \AA$, and six, in pairs, at distances between $2.71 \AA$ and $2.80 \AA^{2}$. The short distance of atomic separation can be looked upon as giving rise to $\mathrm{Ga}_{2}$ molecules. When this structure melts there is a contraction of $3 \%^{3}$ to give a liquid metal which has many features that are free electron like ${ }^{4}$, this free electron behaviour in the liquid state is common to other semimetals, e.g. $\mathrm{Bi}$ and $\mathrm{Sb}$ and the semiconductor, Ge. Diffraction measurements on liquid Ga have been made both in the normal liquid region and supercooled ${ }^{1,5,8}$, and can yield the interference function $I_{\mathrm{Ga}}(\mathrm{K}),[\mathrm{K}=(4 \pi \sin \theta) / \lambda, 2 \theta=$ scattering angle, $\lambda=$ wavelength of incident $\mathrm{x}$-rays]. Although there are differences in detail (see ${ }^{1}$ for comparative table), on all the first main peaks there is a subsidiary maximum or shoulder on the high $\mathrm{K}$ side. This subsidiary maximum is also a feature of the interference functions of $\mathrm{Ge}, \mathrm{Sb}$ and $\mathrm{Bi}$. For $\mathrm{Ge}^{6}$ and $\mathrm{Sb}^{7}$ a model has been proposed which explains the presence of the subsidiary maximum. The essential features of the model are; (1) there are two possible near neighbour distances in the liquid, $r_{\mathrm{AA}}$ and $r_{\mathrm{BB}}$, with $r_{\mathrm{AA}}>r_{\mathrm{BB}},(2)$ the interaction between $\mathrm{A}$ and $\mathrm{B}$ species is the same as that between A species, so $r_{\mathrm{AB}}=r_{\mathrm{AA}}$. Due to the well known lack of resolution in real space information obtained from Fourier transformation of diffraction measurements, comparison of the model with experiment is carried out

Reprint requests to Dr. B. R. Orton, Physics Department, Brunel University, Kingston Lane, Uxbridge, Middx., U.K. in $\mathrm{K}$ space. The model gives a total interference function, $I_{\mathrm{T}}(\mathrm{K})$, which is composed of only two partial interference functions, $I_{\mathrm{AA}}(\mathrm{K})$ and $I_{\mathrm{BB}}(\mathrm{K})$, because, since $r_{\mathrm{AB}}=r_{\mathrm{AA}}, I_{\mathrm{AB}}(\mathrm{K})=I_{\mathrm{AA}}(\mathrm{K})$. It has been shown ${ }^{6}$ that $I_{\mathrm{T}}(\mathrm{K})$ may be written as

$$
I_{\mathrm{T}}(\mathrm{K})=c_{\mathrm{A}}\left(1+c_{\mathrm{B}}\right) I_{\mathrm{AA}}(\mathrm{K})+c_{\mathrm{B}}{ }^{2} I_{\mathrm{BB}}(\mathrm{K}) .
$$

$c_{\mathrm{A}}=$ atomic fraction of the species which has the longest distance of atomic separation.

\section{Model Interference Function Compared with Experiment}

From the very large number of experimental measurements of the diffraction pattern from liquid $\mathrm{Ga}$ it was convenient to choose one which had had tabulated results published. Data presented in this form were available from the work of Page, Saunderson and Windsor ${ }^{8}$, where smoothed values of $I_{\mathrm{Ga}}(\mathrm{Q})$, obtained from neutron diffraction, were presented. Here the neutron diffraction convention of using $Q$ to replace $K$, the magnitude of the momentum transfer vector, has been employed. As in the earlier work ${ }^{6,7}$, in order to obtain agreement between $I_{\mathrm{T}}(\mathrm{K})$ and $I_{\mathrm{Ga}}(\mathrm{Q})$ the partial interference functions used in Eq. (1) were assumed to be given by Ashcroft and Lekner ${ }^{9}$ hard sphere interference functions, $I(\mathrm{~K}, \sigma, \eta) . \sigma=$ hard sphere diameter and $\eta=$ packing density. $\sigma_{\mathrm{A}}, \sigma_{\mathrm{B}}, \eta_{\mathrm{A}}, \eta_{\mathrm{B}}$ and $c_{\mathrm{A}}$ were all varied in a systematic way until the best possible agreement was obtained with $I_{\mathrm{Ga}}(\mathrm{Q})$. The final values for the parameters were, $\sigma_{\mathrm{A}}=2.78 \AA, \sigma_{\mathrm{B}}=$ $2.25 \AA, \eta_{\mathrm{A}}=0.50, \eta_{\mathrm{B}}=0.47, c_{\mathrm{A}}=0.49$. From Fig. 1 it can be seen that very good agreement has been achieved. In fact, in the region of the main and subsidiary peaks agreement is better than the $5 \%$ suggested ${ }^{8}$ for the error in the experimental measure-

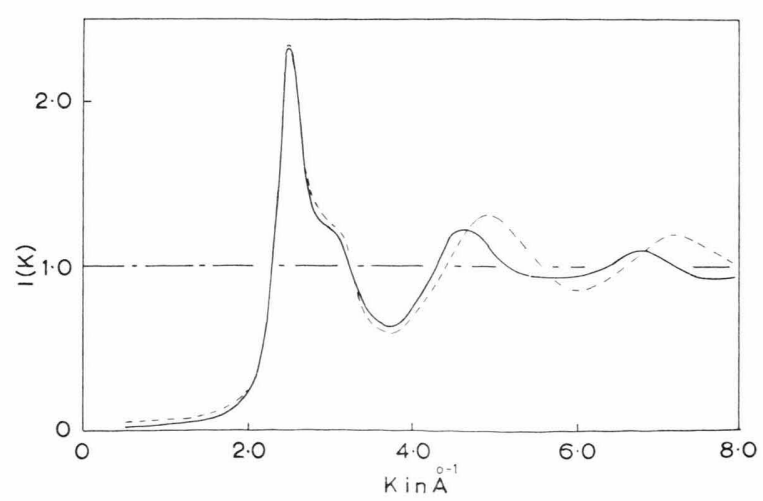

Fig. 1. Interference Functions. - - $-I_{\mathrm{Ga}}(Q)$ at $23^{\circ} \mathrm{C}$ by Page et al. ${ }^{8}$. $I_{\mathrm{T}}(K), \quad \sigma_{\mathrm{A}}=2.78 \AA, \quad \sigma_{\mathrm{B}}=2.25 \AA$, $\eta_{\mathrm{A}}=0.50, \eta_{\mathrm{B}}=0.47$. 
ments. For this reason tabulated values of $I_{\mathrm{T}}(\mathrm{K})$ together with $I_{\mathrm{Ga}}(\mathrm{Q})$ between $\mathrm{K}=1.0 \AA^{-1}$ and $3.8 \AA^{-1}$, are presented in Table 1 , for the purpose

Table $1 . I_{\mathrm{Ga}}(Q)$ and $I_{\mathrm{T}}(K)$ compared.

\begin{tabular}{llllll}
\hline $\begin{array}{l}K \text { or } Q \\
\text { in } \AA^{-1}\end{array}$ & $I_{\mathrm{Ga}}(Q)^{8}$ & $I_{\mathrm{T}}(K)$ & $\begin{array}{l}K \text { or } Q \\
\text { in } \AA^{-1}\end{array}$ & $I_{\mathrm{Ga}}(Q)^{8}$ & $I_{\mathrm{T}}(K)$ \\
\hline 1.0 & 0.06 & 0.03 & 2.6 & 2.08 & 2.09 \\
1.2 & 0.07 & 0.04 & 2.7 & 1.60 & 1.61 \\
1.4 & 0.09 & 0.05 & 2.8 & 1.42 & 1.34 \\
1.6 & 0.12 & 0.08 & 2.9 & 1.31 & 1.25 \\
1.8 & 0.16 & 0.13 & 3.0 & 1.26 & 1.24 \\
2.0 & 0.25 & 0.25 & 3.1 & 1.22 & 1.16 \\
2.1 & 0.33 & 0.37 & 3.2 & 1.09 & 1.01 \\
2.2 & 0.53 & 0.60 & 3.3 & 0.87 & 0.86 \\
2.3 & 0.98 & 1.03 & 3.4 & 0.70 & 0.75 \\
2.4 & 1.68 & 1.74 & 3.6 & 0.59 & 0.65 \\
2.5 & 2.32 & 2.30 & 3.8 & 0.60 & 0.66 \\
\hline
\end{tabular}

of comparison. It is worth commenting that the sign of $\{$ (hard sphere diameter) - (interatomic distance in the crystal structure) $\}$ is negative. Differences of the same sign were found with earlier applications of the model ${ }^{6,7}$, and agree, at least qualitatively, with the volume contraction found on melting. In the two earlier applications of the model another common factor was the packing density of 0.41 used for the hard sphere interference functions. However, in the present model values of 0.51 and 0.47 had to be used. This is possibly because measurements on both $\mathrm{Ge}$ and $\mathrm{Sb}$ were made at temperatures above their freezing point, while measurements on $\mathrm{Ga}$ were made at $23{ }^{\circ} \mathrm{C}$, below the normal freezing point of $29.8^{\circ} \mathrm{C}$.

\section{Discussion}

It appears from the work in this note that the double hard sphere model can be applied to $\mathrm{Ga}$ as well as $\mathrm{Ge}$ and $\mathrm{Sb}$. Following this extension it is appropriate to discuss any theoretical model which can be related to the assumptions on which the present work is based. Recently Silbert and Young ${ }^{10}$ have proposed a model potential for liquid semimetals which takes the form $v(r)=(\infty ; \varepsilon, 0)$ as $(r<\sigma ; \sigma<r<\varrho \sigma ; \varrho \sigma<r), \varepsilon=k_{\mathrm{B}} T_{\mathrm{m}}, \varrho=$ a parameter of the model. The important feature is the ledge in the potential for $\sigma<r<\varrho r$, which introduces the possibility of two hard sphere diameters.

1 A. Bizid, L. Bosio, H. Curien, A. Defrain, and M. Dupont, Phys. Stat. Sol. (a) 23, 135 [1974].

2 R. W. Wyckoff, Crystal Structures, 2nd Ed., Interscience, New York 1965, p. 22.
Silbert and Young have applied their potential in the calculation of $I(K)$ for liquid $\mathrm{Bi}$ and they have been able to reproduce the main features of the experimental $I_{\mathrm{Bi}}(K)$, including the subsidiary maximum on the main peak. It is therefore clear that such a potential could apply to the liquid metals $\mathrm{Ge}, \mathrm{Sb}$ and $\mathrm{Ga}$. Without entering into detailed calculations, one factor which can be compared directly is the ratio of large to small hard sphere diameters for the present model with $\varrho_{\mathrm{Bi}}=2.1$ used by Silbert and Young ${ }^{10}$. The present model gives lower values, $\varrho_{\mathrm{Ge}}=1.30, \varrho_{\mathrm{Sb}}=1.33$ and $\varrho_{\mathrm{Ga}}=1.24$, but they are in better agreement with $\varrho=1.2$ obtained from the length of the ledge for a model potential calculated for $\mathrm{Zn}$, quoted in ${ }^{10}$. Additional evidence for a lower value of $\varrho$ comes from the solid state, where the ratio of the largest to shortest interatomic distances is 1.14. Thus it appears that the model used in the present work can be linked directly to a plausible interatomic potential, though the numerical details do not appear to agree.

Additional theoretical justification for the present model was obtained by considering an explaination put forward by Heine and Weaire ${ }^{11}$ for the structural behaviour of both solid and liquid metals. They suggest that it is energetically unfavourable for strong diffractions in $K$ space to coincide with $K_{0}$, for which the pseudopotential $U\left(K_{0}\right)=0$. If this view is applied in the case of $\mathrm{Ge}, \mathrm{Sb}$ and $\mathrm{Ga}$ it would be expected that the peaks of the partial interference functions, $I_{\mathrm{AA}}\left(K_{\mathrm{A}}\right)$ and $I_{\mathrm{BB}}\left(K_{\mathrm{B}}\right)$ would not fall at $K$ values close to $K_{0}$. Since the first peak of $I_{\mathrm{T}}(K)$ spans the region about $K_{0}$ it would be expected that $K_{\mathrm{A}}<K_{0}<K_{\mathrm{B}}$. This can be shown to be the case for $\mathrm{Ge}\left(2.5 ; 2.9^{12} ; 3.2\right), \mathrm{Sb}\left(2.1 ; 2.8^{12} ; 2.9\right)$ and $\mathrm{Ga}\left(2.5 ; 2.7^{12} ; 3.1\right)$, where the numbers in the brackets are in the sequence of the inequality (units, $\left.\AA^{-1}\right)$.

The same explaination as employed in the last paragraph may be taken further and can account, in a qualitative way, for the second assumption used in the double hard sphere model, i.e. $I_{\mathrm{AB}}(K)=I_{\mathrm{AA}}(K)$. This implies that the first peak position of $I_{\mathrm{AB}}\left(K_{\mathrm{AB}}\right)$ also avoids $K_{0}$. Therefore this is the reason why A and $\mathrm{B}$ species are not present in the liquid as a random mixture. Because, if they were, $K_{\mathrm{AB}}$ would fall between $K_{\mathrm{A}}$ and $K_{\mathrm{B}}$ and, hence, close to $K_{0}$. Although this approach using the pseudopotential is very attractive, it must be mentioned that the basis of the method has been criticised, details can be found in ${ }^{13}$.

3 O. R. Kubaschewski, Trans. Faradey Soc., 45, 931 [1949].

4 T. E. Faber, The Physics of Metals, (Ed. J. M. Ziman), University Press, Cambridge 1969, p. 284. 
5 D. G. Carlson, J. Feder, and A. Segmüller, Phys. Rev. A 9, 400 [1974]

${ }^{6}$ B. R. Orton, Z. Naturforsch. 30 a, 1500 [1975].

7 B. R. Orton, Z. Naturforsch. 31 a, 397 [1976].

8 D. I. Page, D. H. Saunderson, and C. G. Windsor, J. Phys. C: Solid State Phys. 6, 212 [1973].

9 N. W. Ashcroft and J. Lekner, Phys. Rev. 145, 83 [1966].

10 M. Silbert and W. H. Young, Phys. Letters 58 A, 469 [1976].
11 V. Heine and D. Weaire, Solid State Phys. (Ed. H. Ehrenreich, F. Seitz, and D. Turnbull), Academic Press, New York, 24, 455 [1970].

12 M. Cohen and V. Heine, Solid State Phys. (Ed. H. Ehrenreich, F. Seitz, and D. Turnbull), Academic Press, New York, 24, 235 [1970].

13 T. E. Faber, An Introduction to the Theory of Liquid Metals, University Press, Cambridge 1972, p. 133. 